Sociologie et sociétés

\title{
Le « Mode 2 » et la globalisation des systèmes d'innovation « nationaux »
}

\section{Le modèle à Triple hélice des relations entre université, industrie et gouvernement}

\section{Loet Leydesdorff et Henry Etzkowitz}

Volume 32, numéro 1, printemps 2000

La science. Nouvel environnement, nouvelles pratiques?

URI : https://id.erudit.org/iderudit/001434ar

DOI : https://doi.org/10.7202/001434ar

Aller au sommaire du numéro

Éditeur(s)

Les Presses de l'Université de Montréal

ISSN

0038-030X (imprimé)

1492-1375 (numérique)

Découvrir la revue

Citer cet article

Leydesdorff, L. \& Etzkowitz, H. (2000). Le « Mode 2 » et la globalisation des systèmes d'innovation " nationaux " : le modèle à Triple hélice des relations entre université, industrie et gouvernement. Sociologie et sociétés, 32(1), 135-156. https://doi.org/10.7202/001434ar d'utilisation que vous pouvez consulter en ligne. 


\title{
Le «Mode 2» et la globalisation des systèmes d'innovation «nationaux"
}

\author{
le modèle à Triple hélice \\ des relations entre université, \\ industrie et gouvernement
}

\author{
LOET LEYDESDORFF \\ Department of Science and Technology Dynamics \\ University of Amsterdam \\ OZ Achterburgwal 237 \\ 1012 DL Amsterdam \\ The Netherlands \\ Courriel: loet@leydesdorff.net
}

\author{
HENRY ETZKOWITZ \\ Science Policy Institute \\ Social Science Division \\ State University of New York at Purchase \\ 735 Anderson Hill Road \\ Purchase, NY 10577-1400, USA \\ Courriel : HenryEtzkowitz@earthlink.net
}

Traduction : Suzanne Mineau

$\mathrm{O}$ $\mathrm{n}$ a récemment proposé trois modèles pour expliquer les relations socio-économiques dans l'infrastructure de production: (1) le modèle des systèmes d'innovation nationaux (voir par exemple, Lundvall, 1988 et 1992; Nelson, 1993); (2) le modèle «Mode 2 » émergeant de la production de la connaissance scientifique (Gibbons et coll., 1994) ; enfin (3) le modèle de la Triple hélice des relations entre l'université, l'industrie et le gouvernement (Etzkowitz et Leydesdorff, 1995, 1997 et 2000).

Le modèle de la Triple hélice améliore le modèle des systèmes d'innovation nationaux en faisant une variable de la «fonction gouvernementale». De cette façon, il est possible d'étudier dans un cadre cohérent les différents niveaux de gouvernement (européen, national, régional et local). Comme le modèle «Mode 2 », la Triple hélice se concentre sur la couverture dynamique formée par les négociations et les alliances entre les porteurs institutionnels. L'information est sans cesse codifiée en nouvelles connaissances quand elle est communiquée de façon réflexive à l'intérieur de chaque hélice par récursivité et entre les hélices par interaction. Les flux de connaissance et d'information deviennent un nouveau mécanisme de coordination de la société, en parallèle et en interaction avec les relations d'échanges (économiques) et les mécanismes de contrôle (politiques) qui existent déjà. L'économie politique tend ainsi à se fonder de plus en plus sur la connaissance. 
On peut s'attendre à ce que trois dynamiques interactives (production de connaissances, marchés, c'est-à-dire diffusion et contrôle) génèrent des configurations non linéaires renfermant tous les comportements cahotiques possibles (crises, formation de trajectoires, bifucations, etc.). Il est nécessaire de disposer d'un modèle algorithmique pour analyser des dynamiques aussi complexes. De plus, selon le principe de «la Triple hélice», dans une économie fondée sur la connaissance, l'université est en mesure de jouer un rôle élargi en matière d'innovation. Du point de vue analytique, le modèle est différent du modèle des systèmes nationaux d'innovation, qui considère que l'entreprise joue le premier rôle en innovation, et du «triangle» de Sabato (1975) qui privilégie l'État (voir Sabato et Mackenzie, 1982). Nous nous concentrons sur la couverture des réseaux de communications et d'anticipations qui refaçonnent les arrangements institutionnels entre les universités, les entreprises et les organismes gouvernementaux. Nous partons de l'hypothèse que l'université joue un rôle potentiellement prédominant puisque la fonction de production de connaissances est de plus en plus intégrée dans l'infrastructure de la connaissance (Godin et Gingras, 2000).

\section{PRÉDOMINANCE DE LA RECHERCHE UNIVERSITAIRE}

Avec la réduction du rôle des militaires et la place plus importante prise par les milieux universitaires dans les structures institutionnelles des sociétés contemporaines, le réseau des relations universitaires, industrielles et étatiques s'est également transformé; le mode trilatéral de «l'élite de pouvoir» de la guerre froide proposé par Wright Mills (1958) a fait place à une couverture de communications réflexives. Comme les relations entre l'université, l'industrie et le gouvernement ne sont plus hiérarchiquement organisées dans une société multiforme, les effets de cette transformation font l'objet d'un débat international sur le rôle de l'université dans le transfert de la technologie et de la connaissance.

C'est ainsi qu'un rapport suédois sur la recherche en l'an 2000 a recommandé le retrait des universités du projet de «la troisième mission» qui prévoyait leur collaboration directe avec les entreprises. L'université devait plutôt revenir aux tâches de recherche et d'enseignement telles qu'on les concevait traditionnellement (voir Benner et Sandström, 2000). Toutefois, on peut s'attendre à ce que les défenseurs de la troisième mission, qui sont des universités nouvelles et des collèges régionaux et qui ont fondé leurs programmes de recherche sur les prémisses de cette troisième mission, continuent de défendre leur cause. La science et la technologie jouent un rôle important dans le développement régional (voir par exemple Saxenian, 1994; Cooke et coll., 1997; Braczyk et coll., 1998). Il devient aussi possible d'analyser aussi bien une entreprise comme Rand que les institutions d'enseignement supérieur du point de vue des marchés (Dasgupta et David, 1994).

Les questions débattues en Suède ont trouvé un écho aux États-Unis dans les critiques que plusieurs économistes (par exemple, Rosenberg et Nelson, 1994) ont fait du transfert de la technologie universitaire. Ils soutiennent que les mécanismes de transfert risquent de créer des coûts de négociation inutiles en enfermant dans des brevets des connaissances qui autrement circuleraient librement. On peut se demander, par contre, si les connaissances seraient efficacement transmises à l'industrie sans l'existence de la 
série de mécanismes qui identifient et accroissent les domaines d'application des résultats des recherches. Peut-on pousser les processus de développement plus loin en octroyant des subventions spéciales ou en créant de nouvelles entreprises sur les campus et dans les incubateurs universitaires?

Les innovations institutionnelles visent à promouvoir des relations étroites entres les facultés et les entreprises. "La frontière infinie» de la recherche fondamentale, financée comme une fin en soi et avec l'attente lointaine de résultats pratiques, est remplacée par un modèle de «transition infinie» dans lequel la recherche fondamentale est reliée à son utilisation par une série de processus intermédiaires (Callon, 1998) souvent parrainés par l'État. Le modèle linéaire, qu'il reproduise «la force de traction du marché» ou «la force de poussée de la technologie», ne réussit plus à provoquer un transfert de la connaissance et de la technologie. Les publications et les brevets ont des cadres de référence différents les uns par rapport aux autres et aussi par rapport à la transformation de la connaissance et de la technologie en produits commercialisables. Il faut réécrire les règles et réglementations et inventer une stratégie d'interface pour intégrer «la force de traction du marché» et «la force de poussée de la technologie» grâce à de nouveaux mécanismes organisationnels (par exemple, OCDE, 1980; Rothwell et Zegveld, 1981).

Aux États-Unis, ces mécanismes englobent le programme Small Business Innovation Research et le programme Small Business Technology Transfer du département de la défense, ainsi que les Industry/University Cooperative Research Centers et les Engineering Research Centers de la National Science Foundation, etc. (Etzkowitz et coll., 2000). En Suède, on a fait d'une Fondation dédiée à la connaissance et d'une autre dédiée à la technologie deux sociétés d'État à capital de risque financées par un Fonds des salariés qui devait à l'origine servir à l'achat d'actions d'entreprises bien établies. Le mouvement amorcé en Suède pour orienter devantage les universitaires dans cette même direction a provoqué un débat similaire à celui qui a eu lieu aux États-Unis au début des années 1980. Au même moment, l'université Harvard cherchait à créer une entreprise conjointement avec l'un de ses professeurs, à partir des résultats de ses recherches.

Les milieux universitaires peuvent-ils remplir cette troisième mission de développement économique, en plus de leurs missions de recherche et d'enseignement? Comment chacune de ces tâches peut-elle contribuer à la mission de l'université? À la fin du $19^{\mathrm{e}}$ siècle, il s'est produit une révolution dans les universités lorsqu'on a ajouté la recherche à leur mission et qu'on l'a rendue plus ou moins compatible avec l'enseignement, du moins au niveau supérieur. Beaucoup d'universités des États-Unis et d'ailleurs dans le monde sont encore en train d'assimiler cette transformation. La place de plus en plus prédominante que prennent la connaissance et la recherche dans le développement social et économique a fait naître une troisième mission: le rôle de l'université dans le développement socio-économique. Une «deuxième révolution universitaire» semble se dérouler depuis la Seconde Guerre mondiale, mais elle a acquis davantage de visibilité depuis la fin de la guerre froide (Etzkowitz, à paraître).

Aux États-Unis dans les années 1970, dans divers pays d'Europe de l'Ouest dans les années 1980 et en Suède aujourd'hui, cette transition entraîne une réévaluation de la 
mission et du rôle de l'université dans la société. Des débats similaires ont lieu en Amérique latine, en Asie et ailleurs en Europe. Une série de conférences sur «la Triple hélice» (Amsterdam, 1996; Purchase NY, 1998 et Rio de Janeiro, 2000) a donné aux universitaires et aux analystes politiques l'occasion de discuter de questions théoriques et empiriques (Leydesdorff et Etzkowitz, 1996 et 1998). Différentes configurations possibles des relations entre les shères institutionnelles universitaires, industrielles et étatiques peuvent contribuer à générer d'autres stratégies de croissance économique et de transformation sociale.

TROIS CONFIGURATIONS DE LA TRIPLE HÉLICE

L'évolution des systèmes d'innovation et le conflit actuel au sujet du chemin que doivent emprunter les relation entre l'université et l'industrie se reflètent dans les différents arrangements institutionnels qui déterminant les relations entre les universités, les entreprises et les gouvernements. D'un point de vue analytique, ces diverses configurations peuvent être considérées comme des «instants» (Giddens, 1984) du modèle plus général de la Triple hélice. Ces modèles politiques peuvent jouer le rôle de guide normatif et politique.

Premièrement, il peut exister une situation historique précise que nous appelons «la Triple hélice». Dans cette configuration l'État nation encercle les entités universitaires et industrielles et dirige les relations entre elles (Figure 1). On peut trouver une forme évidente de ce modèle dans l'ancienne unss et dans certains pays d'Europe de l'Est sous leur « régime socialiste actuel. Des versions plus floues se retrouvent dans les politiques de beaucoup de pays d'Amérique latine et, jusqu'à un certain point, dans des pays européens comme la Norvège.

Un second modèle politique (Figure 2) est formé de sphères institutionnelles distinctes ayant entre elles des frontières très nettes et des relations très circonscrites. On le retrouve en Suède dans le rapport sur la recherche mentionné précédemment ainsi qu'aux États-Unis dans l'opposion qu'ont suscitée les divers rapports de la GovernmentUniversity-Industry Research Roundtable du Conseil national de recherche (MacLane, 1996; voir GUIRR, 1998). Finalement, le modèle le plus poussé, «la Triple hélice III », devrait générer une infrastructure de la connaissance dans laquelle les sphères institutionnelles se chevauchent, chacune assumant le rôle de l'autre, ce qui donne naissance à des organismes hybrides aux interfaces (Figure 3). La couverture intègre partiellement les arrangements sous-jacents, mais ils sont répartis en mode réseau. Le système demeure incomplet et, par conséquent, en changement continuel.

Les différences entre les deux premières configurations des relations entre l'université, l'industrie et le gouvernement suscitent actuellement un intérêt normatif. On considère généralement que «la Triple hélice I» est un échec en tant que modèle de développement. À cause du manque d'espace pour que des initiatives circulent «de bas en haut», l'innovation s'est trouvée étouffée au lieu d'être stimulée. «La Triple hélice II » suppose une politique du laisser-faire qui est pronée aujourd'hui comme thérapie de choc dans le modèle de «la Triple hélice I» afin de réduire le rôle de l'État. La plupart 
des pays et régions tentent actuellement de réaliser, sous une forme ou une autre, le modèle complet, «la Triple hélice III».

L'objectif commun est de créer un environnement innovateur dans lequel on retrouve des entreprises dérivées de l'université, des initiatives trilatérales de développement économique fondé sur la connaissance ainsi que des alliances entre des entreprises (petites et grandes) œuvrant dans des secteurs distincts et à des niveaux technologiques différents, des laboratoires gouvernementaux et des groupes de recherche universitaires. L'État n'impose pas ces arrangements, mais les favorise souvent, que ce soit au moyen de nouvelles «règles du jeu », d'une aide financière directe ou indirecte, de la loi BayhDole aux États-Unis ou de l'introduction de nouveaux acteurs comme les fondations créées en Suède pour promouvoir l'innovation.

\section{LE MODÈLE À TRIPLE HÉLICE DES IN NOVATIONS}

En tant que modèle analytique, la Triple hélice complète la description des divers arrangements institutionnels et modèles politiques par une explication de leur dynamique. Lorsqu'un système d'innovation est mis sur pied, quelles sont les unités opératoires qui interagissent? Comment un système aussi complexe peut-il être spécifique?

À notre avis, des modèles types comme «les systèmes nationaux d'innovation " (Lundvall, 1988; Nelson, 1993), "les systèmes de recherche en transition» (Cozzens et coll., 1990; Ziman, 1994), le «Mode 2» (Gibbons et coll., 1994) ou le «système de recherche post-moderne» (Rip et Van der Meulen, 1996) indiquent tous un changement perpétuel, une réorganisation et le rôle accru de la connaissance dans l'économie et la société. Pour pouvoir expliquer ces réorganisations observables dans les relations entre université, industrie et gouvernement, il est nécessaire de transformer les théories sociologiques sur le rôle de conservation des institutions, sur l'innovation par recombinaisons et sur les contrôles réflexifs. Chaque théorie devrait servir à évaluer une sous-dynamique différente (Leydesdorff, 1997).

Contrairement à une double hélice (ou à une coévolution entre deux dynamiques), une triple hélice ne peut pas générer à long terme de la stabilité. La métaphore biologique ne vaut plus à cause de la différence entre l'évolution culturelle et l'évolution biologique. Dans la théorie de l'évolution biologique, les différences constituent la force motrice, et la sélection se fait naturellement. L'évolution culturelle, par contre, est provoquée par des décisions conscientes de la part d'individus et de groupes ainsi que par l'apparition de conséquences non intentionnelles. On peut s'attendre à ce qu'une triple hélice, dans laquelle chaque brin peut être relié aux deux autres, développe une couverture réflexive de communications, de réseaux et d'organisations entre les hélices. Ce résultat peut être considéré comme une conséquence non intentionnelle d'un développement sociétal qui n'exige plus de réalignement de la part d'un gouvernement central (comme en temps de guerre).

Dans une configuration à Triple hélice, on ne peut plus penser que les sources des innovation sont synchronisées a priori. Elles ne s'imbriquent plus les unes dans les autres selon un ordre préétabli, mais elles génèrent des casse-tête que les participants, 
les analystes et les décideurs politiques doivent résoudre. Ce réseau de relations a un effet rétroactif sur les intentions, les stratégies et les projets; cette rétroaction ajoute une valeur excédentaire en réorganisant et en harmonisant sans cesse l'infrastructure sousjacente pour obtenir au moins une approximation des divers objectifs. Dans la mesure où ces dynamiques sont contrôlées, il devient possible de préciser un programme de recherche axé sur l'innovation.

Les systèmes d'innovation et leurs relations entre eux apparaissent aux niveaux organisationnel, local, régional, national et multi-national. Les sous-dynamiques interactives, c'est-à-dire des éléments opératoires spécifiques comme les marchés et les innovations technologiques, sont constamment reconstruites à la manière du commerce sur internet, mais de façon différente aux divers niveaux. Une reconstruction réflexive de ces sous-dynamiques et des niveaux se fait aussi au moyen des discussions et des négociations. On ne peut considérer comme allant de soi et réifier ce qui est «industrie» et ce qui est «marché». Chaque "système» est défini et peut être redéfini en fonction de la conception du projet de recherche.

Par exemple, des «systèmes nationaux d'innovation» peuvent être plus ou moins systémiques. Par contre, leur degré de systémisation demeure une question empirique. (Voir dans Leydesdorr et Oomes (1999) un test du systémique.) Un «système d'innovation» dynamique peut englober des liens de collaboration de plus en plus complexes au-delà des frontières nationales et entre des chercheurs et des utilisateurs de recherche de diverses sphères institutionnelles. Il peut y avoir des dynamiques différentes parmi les régions (Cooke, 1998; Riba-Vilanova et Leydesdorff, 1999). Il faut préciser les cadres de référence sur un plan analytique, c'est-à-dire en tant qu'hypothèses soutenues par une théorie. La Triple hélice vient ajouter une autre hypothèse: les arrangements seront transitoires. Les observations donnent l'occasion de corriger les anticipations analytiques.

\section{UNE TRANSITION INFINIE}

L'infrastructure des économies fondées sur la connaissance suppose une transition infinie. La grande vision de Marx selon laquelle «tout ce qui est solide se dissout dans l'air» (Berman, 1982) sous-estimait l'importance des communications et interactions, volatiles en apparence, dans la recodification du système (complexe) de réseaux. Lorsque la connaissance est utilisée de plus en plus comme une ressource par les systèmes de production et de distribution, la reconstruction qui prévaut peut être un mode de «destruction créatrice» (Schumpeter, 1939 et 1966; Luhmann, 1984).

Les forces de reconstruction peuvent-elles être spécifiées? Il existe un mode de spécification dans un système économique évolutif doté des trois mécanismes fonctionnels suivants: l'innovation technologique crée de la diversité, ce sont surtout les marchés qui font la sélection et les structures institutionnelles assurent la conservation et le contrôle réflexif du système (Nelson, 1994). Dans des sociétés avancées et multiformes, les mécanismes de contrôle institutionnel se différencient encore davantage entre les secteurs public et privé. Il se développe donc un système complexe constamment soumis à l'intégration et à la différenciation, aux niveaux à la fois local et global. 
Dans cette dynamique complexe, on peut définir l'innovation à différents niveaux et à partir de points de vue différents. Par exemple les partisans d'un système économique évolutif ont soutenu que les entreprises doivent constituer les unités d'analyse puisqu'elles sont les porteuses de l'innovation et qu'elles doivent se faire concurrence sur les marchés (Nelson et Winter, 1982; voir Andersen, 1994). D’un point de vue politique, on pourrait vouloir faire du «système national d'innovation» le cadre de référence approprié des interventions gouvernementales. D'autres favorisent les réseaux parce qu'ils constituent des unités d'analyse plus abstraites: leur dynamique semi-autonome peut indiquer des blocages, une coupure, etc. (par exemple, David et Foray, 1994). En outre, des réseaux en évolution peuvent modifier leurs frontières en se développant (Maturana, 1978).

À notre avis, ces différents points de vue ouvrent des fenêtres pour évaluer les processus dynamiques et complexes de l'innovation, mais sous des angles spécifiques. Cette dynamique complexe comporte des sous-dynamiques comme les forces du marché, le pouvoir politique, le contrôle institutionnel, les mouvements sociaux, les trajectoires et les régimes technologiques. On peut s'attendre à ce que les éléments opératoires soient emboîtés et interactifs. Par exemple, à l'intérieur d'une grande entreprise ou d'un État nation, l'intégration ne peut être considérée comme allant de soi. L'innovation technologique peut aussi exiger la refonte d'un organisme ou d'une collectivité (Freeman et Perez, 1988). Par contre, le système n'est pas déterministe: des actions intentionnelles peuvent mieux réussir à orienter le changement technologique dans certaines phases que dans d'autres (Hughes, 1983).

La dynamique est non linéaire, tandis que les éléments interactifs et récursifs doivent être connus. Il y a d'abord des transformations constantes à l'intérieur de chacune des hélices. On peut considérer ces reconstructions comme un niveau d'innovations continues sous la pression de changements dans l'environnement. Lorsqu'il y a une adaptation conformationnelle mutuelle entre deux hélices, leur coévolution peut entraîner une stabilisation le long d'une certaine trajectoire. Si plus d'une interface est stable, on peut prévoir la formation d'un régime global. Les cycles générés à chaque niveau guident les phases du développement. Les transformations d'un ordre supérieur (à plus long terme) sont provoquées par celles d'un ordre inférieur, mais ces dernières peuvent être profondément perturbées par des événements d'un ordre adjacent (Schumpeter, 1939; Kampmann et coll., 1994).

Même s'il est abstrait, le modèle nous permet de préciser les diverses fenêtres d'évaluation théorique sous l'angle des sous-dynamiques constitutives (voir Leydesdorff et Van den Besselaar, 1997). Ces diverses sous-dynamiques se sélectionnent mutuellement de façon asymétrique, comme dans les processus de négociation, en utilisant leurs codes spécifiques. Par exemple, les marchés et les réseaux font leur sélection en fonction de la faisabilité technologique, tandis que les options d'avancées technologiques peuvent aussi être précisées en fonction des forces du marché. Les gouvernements peuvent intervenir en collaborant à la création d'un nouveau marché ou, autrement, en changeant les règles du jeu. 
Quand les sélections «se bloquent» entre elles, les systèmes de l'ordre suivant peuvent devenir pertinents. Par exemple, le développement d'un avion au niveau d'une entreprise génère des trajectoires au niveau de l'industrie et il se crée des coévolutions entre les technologies et les marchés qui ont été choisis (McKelvey, 1996). De nos jours, le développement d'une nouvelle trajectoire technologique suppose l'appui des gouvernements nationaux et même internationaux (comme en Europe) et fait de plus en plus appel à un règime à triple hélice (Frenken et Leydesdorff, 2000). Notre approche nous permet de poursuive l'analyse au niveau des réseaux, puis de comparer les configurations des relations. Par exemple, les industries et les gouvernements sont entraînés dans des transformations institutionnelles, tandis que ces transformations elles-mêmes se modifient sous la pression des technologies d'information et de comunication (TIC) ou des politiques gouvernementales. Toutefois, avant d'expliquer les implications du modèle à Triple hélice, nous voulons insister à nouveau sur le caractère analytique du modèle par rapport aux autres modèles non linéaires comme le «Mode 2 » et les «systèmes nationaux d'innovation». Comment un modèle algorithmique, qui fonctionne en se fondant sur des changements relationnels et des anticipations, diffère-t-il des métaphores géométriques fondées sur des observations historiques qui ont prédominé jusqu'à maintenant dans la littérature?

\section{LES MODÈLES D'INNOVATION NON LINÉAIRES}

Les modèles non linéaires de l'innovation vont plus loin que les modèles linéaires parce qu'ils prennent en compte des éléments interactifs et récursifs. Ces éléments non linéaires peuvent modifier les relations causales entre les données d'entrée et de sortie. Dans les systèmes que nous étudions par exemple, on peut s'attendre à ce que les règles de production changent avec le développement plus poussé des relations entrée/sortie (par exemple, à cause des économies d'échelle). Par conséquent, l'unité opératoire peut se transformer, comme cela se produit généralement dans l'industrie chimique lorsqu'une usine pilote devient un établissement de production.

En changeant l'unité d'analyse pour en faire une unité opératoire au niveau réflexif, on obtient une perspective différente du système à l'étude. Par contre, le système luimême évolue. D’un point de vue méthodologique, le modèle conceptuel est remis en question puisqu'il faut pouvoir déterminer si c'est la variable qui a changé ou simplement sa valeur. L'analyse discursive nous fournit un instantané tandis que la réalité nous fournit un film. Pour comprendre l'analyse discursive, il nous faut des métaphores qui réduisent la complexité. Il est possible de stabiliser les métaphores géométriques à l'aide de codifications d'un ordre supérieur, comme dans le cas des paradigmes. Toutefois, pour comprendre les flux (c'est-à-dire la façon dont les variables et leur valeur peuvent changer avec le temps), il faut faire appel à des simulations algorithmiques. Les éléments observables peuvent alors être considérés comme des cas spéciaux qui expliquent les anticipations (Leydesdorff, 1995).

L'innovation en particulier ne peut être définie que sous un angle opératoire. On s'attend à ce qu'elle modifie à la fois les innovateurs et les systèmes d'inovation. En 
outre, il est possible d'être à la fois un observateur et un participant, et aussi de changer de perspective. Dans l'analyse, par contre, les deux rôles sont différenciés même si, dans la «vraie vie», ils peuvent se fusionner. Langton (1989) a proposé de différencier le niveau "phénotypé» des éléments observables et le niveau "génotypé» de la théorie analytique. Il reste à expliquer les "phénotypes», et les diverses explications se font concurrence du point de vue clarté et utilité au moment de corriger les anticipations. Il est difficile d'éviter la confusion étant donné la pression exercée en faveur de conclusions normatives, alors que les différents points de vue se font sans cesse concurrence, sous l'angle normatif comme sous l'angle analytique.

Regardons d'abord le problème que posent l'unité d'analyse et l'unité opératoire. En plus d'allonger les modèles linéaires (entrée/sortie) des analyses néo-classiques et des analyses d'entreprises à cause de leur perspective récursive, les théoriciens de l'économie évolutive ont également modifié l'unité d'analyse. Alors que les économistes néo-classiques se concentraient sur les marchés et les réseaux du point de vue des relations entrée/sortie entre agents informés et rationnels, les économistes évolutifs ont eu tendance à se concentrer sur les entreprises en tant que porteurs spécifiques et obligés du processus d'innovation. Ils ont donc modifié ainsi l'unité d'analyse et l'unité opératoire (Andersen, 1994; voir Alchian, 1950).

Lundvall (1988, p. 357) fait observer que les éléments interactifs entre la demande et l'offre dans les relations utilisateur-producteur présuppose, à chaque moment dans le temps, un cadre de référence en plus d'un marché. Selon lui, la dispute classique au sujet de la théorie des innovations avait trait au rôle de la demande et de l'offre, c'est-à-dire des forces du marché, dans la détermination du rythme et de l'orientation du processus d'innovation (voir Mowery et Rosenbert, 1979; Freeman, 1982, p. 211). Par contre, pour que la dynamique de l'innovation (c'est-à-dire la concurrence entre les produits) soit différente de la dynamique du marché (c'est-à-dire la concurrence entre les prix), il est nécessaire de préciser pour la sélection un autre cadre de référence par rapport à l'axe temps.

Lundvall (1988) a proposé de "prendre comme point de départ le système de production national pour définir un système d'innovation» (p. 362). Il ajoute cependant qu'il ne faut pas considérer le système de production national comme un système fermé: «le degré spécifique de l'ouverture et la forme qu'elle prend déterminent la dynamique de chaque système national de production». À notre avis, il faudrait considérer dans un premier temps les sytèmes de production comme la dynamique de changement des deux systèmes de production et de distribution. Sous cet angle, les systèmes nationaux se font concurrence quant à l'adaptabilité de leur infrastructure de connaissance. Comment les compétences se répartissent-elles pour résoudre «le casse-tête de la production» dû à des avancées technologiques inégales parmi les secteurs (Nelson et Winter, 1975; Nelson 1982)?

Les processus d'innovation qui peuvent exister à l'intérieur des secteurs ou entre eux dépendent de l'infrastructure. La répartition des acteurs pertinents, notamment, représente un potentiel heuristique qu'une analyse stratégique des forces et des faiblesses spécifiques peut rendre réflexif (Pavitt, 1984). La résolution du casse-tête de la production demande l'intervention gouvernementale dans le modèle, et la dynamique passe 
d'une double à une triple hélice. Les processus de négociations qui s'ensuivent sont à la fois complexes et dynamiques: on peut s'attendre à ce que les interactions entraînent une réplication et un changement des acteurs (institutionnels). Des réseaux trilatéraux et des organismes hybrides se créent pour résoudre les crises sociales et économiques. Les acteurs des différentes sphères négocient et définissent de nouveaux projets, comme l'invention d'une société à capital de risque en Nouvelle-Angleterre au début de l'aprèsguerre (Etzkowitz, à paraître). La dynamique de la Triple hélice des relations entre l'université, l'industrie et le gouvernement intervient donc de façon endogène.

Selon Gibbons et ses collaborateurs (1994), ce «nouveau mode de production de la connaissance scientifique» est évident aujourd'hui. Par contre, comment peut-on considérer que la dynamique des ententes des réseaux industriels, gouvernementaux et universitaires soit une conséquence des interactions utilisateur-producteur que Lundvall (1988) mettait au premier plan? Les systèmes nationaux représent-ils encore une unité d'analyse pertinente? Comme le nouveau mode de production de la connaissance (le «Mode 2») devient un résultat, il faut, selon nous, le considérer comme un système naissant. Ce système naissant repose et se construit comme un hyper-réseau sur les autres réseaux (comme les disciplines, les industries et les gouvernements nationaux), mais l'économie du savoir transforme «le navire pendant qu'une tempête fait rage en mer» (Neurath et coll., 1929).

L'organisation de la science a toujours comporté des réseaux pour la poursuite d'objectifs pratiques ou théoriques. Des siècles avant de se métamorphoser en site internet, Mersenne était un savant qui, par ses rencontres et sa correspondance, unissait la communauté scientifique d'Europe. À partir du 16e siècle, ce fut l'Académie des sciences qui joua ce rôle unificateur au niveau local et national. Le côté pratique des découvertes scientifiques n'est pas récent. Dans sa thèse (1938), Robert K. Merton signalait qu'entre 40 et $60 \%$ des découvertes du $17^{\mathrm{e}}$ siècle étaient issues de tentatives pour résoudre des problèmes de navigation, d'exploitation minière, etc. À l'inverse, la solution de problèmes pratiques à l'aide de méthodes scientifiques a constitué un important facteur de développement des sciences, qu'il s'agisse de la pharmacologie allemande au $17^{\mathrm{e}}$ siècle (Gustin, 1975) ou de la concurrence sur les mers financée par l'Angleterre pour assurer la sécurité de la navigation (Sobel, 1995).

Le soi-disant «Mode 2 » n'est pas nouveau. Il représente la structure originale de la science avant son institutionnalisation dans une académie au $19^{e}$ siècle. On peut aussi se demander pourquoi ce n'est qu'après le «Mode 2» qu'a surgi le «Mode 1», c'est-àdire le fondement organisationnel et institutionnel de la science constitué de réseaux et de collèges invisibles (voir Weingart, 1977; Godin, 1998). D'où vient l'idée selon laquelle les scientifiques sont des personnes isolées et la science, une entreprise n'ayant pas les mêmes intérêts que la société? Le «Mode 2 » représente la base matérielle de la science, son véritable mode de fonctionnement. Le «Mode 1 » est une construction posée sur cette base pour justifier l'autonomie de la science, surtout à une époque antérieure quand elle était encore une institution fragile et qu'elle avait besoin de toute l'aide qu'elle pouvait trouver. 
Aux États-Unis, au cours du $19^{\mathrm{e}}$ siècle, des dons importants ont permis la création de nouvelles universités et l'agrandissement de celles qui existaient déjà. Beaucoup d'universitaires craignaient que les industriels qui faisaient ces dons tentent d'influencer directement les universités en réclamant le droit d'embaucher et de remercier les professeurs et aussi de décider quels sujets convenaient ou non à la recherche et à l'enseignement (Storr, 1953). Afin de donner à la science un espace indépendant, à l'abri du contrôle des intérêts économiques, le physicien Henry Rowland se mit à prôner la doctrine suivante: l'intervention de toute personne ayant des intérêts étrangers à la science nuisait à la conduite des travaux scientifiques. À la fin du $19^{\mathrm{e}}$ siècle, devenu président de la American Association for the Advancement of Science, il fit la promotion de l'idéologie de la recherche pure. En même temps qu'étaient fondées des universités libérales dédiées à la recherche pure, l'État fédéral accordait des terres à des universités, dont MIT, axées sur la recherche pratique. Ces deux types opposés d'universités ont vécu en parallèle pendant bien des années.

Des décennies plus tard, en 1942, Robert K. Merton a énoncé les principes normatifs de la science et renforcé l'idéologie de la «science pure». En mettant l'accent sur l'universalisme et le scepticisme, il réagissait à une situation historique particulière, soit la nécessité d'empêcher la science d'être corrompue par les préjugés raciaux de la doctrine nazie et par les attaques de la génétique de Lysenko en unss Pour protéger l'espace indépendant de la science, Merton a défini une série de normes qui ont servi de fondement, pendant de nombreuses années à une sociologie empirique de la science.

Le troisième élément qui a contribué à l'enracinement de l'idéologie de la science pure a évidemment été le Rapport Bush en 1945. L'immense réussite de la science pendant la Seconde Guerre mondiale pour trouver des solutions pratiques avait en quelque sorte légitimé son existence. La fin de la guerre approchant, il fallait s'assurer qu'elle continuerait d'être financée en temps de paix en lui trouvant une nouvelle raison d'être. C'est pourquoi, en 1944, Bush persuada le président Roosevelt de commander un rapport à ce sujet (Bush, 1980).

Dans la première version de ce rapport, Bush proposait de financer la science dans les universités en utilisant une méthode courante en Grande-Bretagne. Les fonds devaient être répartis en fonction du nombre d'étudiants dans chaque établissement. Comme le système britannique contemporain comportait un nombre restreint d'universités, les fonds allaient automatiquement à l'élite. Toutefois, si les États-Unis avaient adopté ce modèle, le financement se serait fait de façon différente, même au début de l'après-guerre. Il n'aurait pas avantagé en premier lieu uniquement l'élite universitaires des côtes Est et Ouest, mais il aurait été distribué dans le large éventail des universités, notamment dans les universités populeuses des États du Midwest.

Pendant le temps qui s'écoula entre la première version et la version finale du rapport, on modifia le mode de répartition des fonds gouvernementaux pour la recherche universitaire et on introduisit le système d' "évaluation par les pairs» adapté des pratiques des fondations dans les années 1920 et 1930 . On pouvait s'attendre à ce que «les pairs», c'est-à-dire les scientifiques reconnus qui seraient sûrement membres des 
comités d'évaluation, distribuent les fonds surtout à l'élite scientifique. Cela vint renforcer le système de classement des universités selon leur statut qui existait depuis les années 1920.

Aux yeux de beaucoup, ce modèle de «la meilleure science» ne peut plus constituer la seule base de répartition des fonds publics en recherche. Des membres du Congrès, les représentants de régions dotées d'universités qui ne reçoivent pas beaucoup de fonds de recherche, ont fait fi de l'évaluation par les pairs et se sont mis à distribuer directement des fonds de recherche en utilisant des crédits fédéraux; ce financement fait souvent partie, comme les routes et les ponts, des magouilles politiques appelées «donnant-donnant» ou «partage de l'assiette au beurre». Il n'en demeure pas moins que ces fonds publics financent de la recherche scientifique sérieuse et l'acquisition d'instruments. Même lorsqu'ils sont attribués à des institutions possédant peu d'expérience en recherche ou même aucune, ces «fonds ponctuels» servent généralement aux chercheurs à acquérir rapidement des compétences qui leur permettront de devenir des concurrents dans le système d'évaluation par les pairs.

En fait, lorsqu'une université de pointe comme Columbia a eu besoin de moderniser l'infrastructure de son département de chimie, elle a retenu les services d'une firme de lobbyistes de Washington qui aidait des universités moins connues. Sur les conseils de spécialistes en relations publiques, elle a rebaptisé son département de chimie «centre national d'excellence en chimie». Elle a pu ainsi obtenir des crédits fédéraux spéciaux qui lui ont permis de rénover et d'agrandir ses installations de recherche. Pour garder son corps professoral, elle ne pouvait se permettre de passer par la lente route de l'évaluation des pairs, sans compter qu'elle n'aurait sans doute pas reçu des sommes aussi importantes.

Une concurrence accrue entre les anciens et les nouveaux acteurs pour obtenir des fonds de recherche a entraîné l'apparition de failles dans le système d'évaluation par les pairs qui peut, au mieux, répondre à un nombre modéré de concurrents. Avec l'accroissement constant de la concurrence, comment le système peut-il s'adapter aux tensions? Certains ont proposé une réduction du réseau de recherche; d'autres parlent de lier la science à de nouveaux motifs de légitimité, comme le développement régional.

\section{LA LÉGITIMITÉ DE LA SCIENCE DANS L'AVENIR}

Il apparait clairement aujourd'hui qu'une grande partie du développement industriel de demain dépend de la science. Ces liens entre la science et l'industrie existent pourtant depuis que la science est devenue une activité organisée au cours $\mathrm{du} 17^{\mathrm{e}}$ siècle. Marx les a signalés au milieu du $19^{\mathrm{e}}$ siècle à propos du développement de l'industrie chimique allemande. Parallèlement, il a élaboré une thèse sur la croissance de l'industrie fondée sur la science à partir d'un seul exemple empirique: les recherches de Perkins sur les colorants au Royaume-Uni qui ont entrainé la création d'une industrie en Allemagne.

Au seuil du nouveau millénaire, la contribution potentielle de la science au développement économique suscite de la concurrence entre les régions et les nations. Jusqu’à 
tout récemment, on ne s'inquiétait guère du lieu où se faisait la recherche. On ne voyait pas de liens étroits entre le lieu de production de la connaissance et son lieu d'utilisation éventuelle, même sous l'angle de l'avantage du premier arrivé. Cette façon de voir a dramatiquement changé depuis quelques années, de même que l'idée voulant que des agglomérations de haute technologie, comme la Route 128 et la Silicon Valley, soient des exemples uniques non reproductibles. La progression récente, par exemple, de Austin au Texas est due en partie à l'accroissement des recherches à l'université du Texas grâce à des fonds de l'État, des entreprises et du gouvernemet central.

Des régions où il se fait moins de recherche sont maintenant bien conscientes que la science, appliquée à leurs ressources locales, constitue l'assise d'une grande partie de leur développement socio-économique dans l'avenir. Aux États-Unis, on n'accepte plus que les fonds de recherche soient répartis surtout entre les côtes Est et Ouest avec un peu de soupoudrage dans le Midwest. L'allocation des fonds de recherche se fait par d'autres moyens que celui de l'évaluation par les pairs parce que toutes les régions veulent leur part. La raison classique qui légitimait la recherche scientifique était qu'elle contribuait à la culture; cette raison demeure valable, et il y a également des objectifs militaires et médicaux qui constituent de puissants stimulants. Dans l'avenir pourtant, la raison qui légitimera la recherche scientifique, dotée de fonds très élevés, sera de plus en plus son rôle comme source de nouvelles voies de développement économique.

Des disciplines nouvellement crées sont souvent la raison d'être de cette hausse des attentes. Ces nouvelles disciplines ne représentent pas uniquement des sousdivisions des anciennes comme au $19^{\mathrm{e}}$ siècle (Ben David et Collins, 1966). Tout récemment, de nouvelles disciplines sont nées d'une synthèse d'intérêts pratiques et théoriques. C'est ainsi que les sciences informatiques se sont constituées à partir d'une machine et de segments de disciplines plus anciennes comme le génie électrique, la psychologie et la philosophie. La science des matériaux et d'autres domaines comme la nano-technologie, qui sont sur la liste des technologies essentielles de toutes les nations, ont vu le jour de la même manière.

On peut s'attendre à ce que l'université demeure l'institution au coeur de l'infrastructure de production de connaissance tant qu'elle conservera sa mission éducative originale. L'enseignement lui donne un avantage par rapport aux autres, surtout quand il est relié à la recherche et au développement économique. De plus, les étudiants sont des inventeurs en puissance. Ils représentent un flux dynamique de "capital humain» qui circule sans cesse dans les groupes de recherche universitaires, comparativement aux laboratoires industriels et aux instituts de recherche qui sont plus statiques. Même s'il est parfois considéré comme un dérangement inévitable, le roulement des étudiants assure à l'université sa suprématie comme source des innovations.

Pour assurer le leadership dans le domaine de la connaissance, l'université se compare fort bien à d'autres candidats qui se sont récemment proposés, comme la firme de consultants. Une firme de consultants réunit un personnel d'horizons très dispersés pour un projet précis, puis elle le disperse à nouveau, une fois le projet terminé et le problème particulier d'un client résolu. Ces firmes n'ont pas la capacité organisationnelle 
voulue pour mener un programme cumulatif de recherches dans le cadre de leurs activités normales. Les avantages comparatifs uniques de l'université sont de combiner, grâce au passage des générations d'étudiants, continuité avec changement, organisation et recherche anciennes avec personnes et idées nouvelles. Quand il y a coupure entre les générations, généralement à cause de la disparition de fonds de recherche, un groupe de recherche universitaire disparaît et peut être remplacé par un autre.

Il est vrai que les entreprises organisent de plus en plus des programmes de formation de haut niveau (par exemple Applied Global University de la Applied Materials Devices Corporation, un fabricant de semi-conducteurs de la Silicon Valley); elles pourraient bien dans l'avenir, isolément ou ensemble, tenter aussi de décerner des diplômes. Elles font souvent appel à leur personnel de recherche ainsi qu'à des consultants extérieurs pour dispenser l'enseignement dans leurs propres universités. Toutefois, à l'exclusion de quelques exceptions importantes, comme la Rand Corporation, elles n'ont pas encore réuni systématiquement dans un même cadre la recherche et l'enseignement. Par contre, à mesure que croît la nécessité d'une formation permanente, l'idée de relier l'université au lieu de travail acquiert de l'importance.

\section{IMPLICATIONS DU MODĖLE DE LA TRIPLE HÉLICE}

La Triple Hélice illustre non seulement les relations entre l'université, l'industrie et le gouvernement, mais aussi les transformations à l'intérieur de chacune de ces sphères. Après avoir été au départ une institution d'enseignement, l'université combine maintenant enseignement et recherche; il s'agit d'une révolution qui se poursuit non seulement aux États-Unis, mais dans beaucoup d'autres pays. Il existe des tensions entre les deux activités, mais elles coexistent néanmoins de façon plus ou moins compatible parce que leur combinaison se révèle productive et rentable.

La couverture de la Triple hélice constitue un modèle de la structure sociale qui explique l'apparition au cours de l'histoire du modèle "Mode 2 » et sa relation au «Mode 1». Premièrement, il n'est plus nécessaire de conceptualiser les arrangements industriels et gouvernementaux exclusivement entre des États et des secteurs industriels spécifiques. Des alliances stratégiques transversales apparaissent dans les divisions traditionnelles entre les secteurs; quant aux gouvernements, ils peuvent intervenir au niveau national, régional et aussi, de plus en plus, international. Les entreprises adoptent des positions «globales», soit à l'intérieur de leur propre structure organisationnelle, soit en nouant des alliances. Des blocs commerciaux comme le Marché commun, NAPIA et Mercosul fournissent de nouvelles occasions de rompre les «blocages» sans sacrifier les avantages concurrentiels des anciennes constellations. C'est ainsi qu'on peut considérer l'Airbus comme une opportunité interactive de recombinaison au niveau supranational (Prenken, 2000).

Deuxièmement, il est possible de faire de l'anticipation des profits la force motrice des interactions. Le mot «profit» peut prendre différentes significations selon les acteurs en cause. Ainsi un consommateur à l'avant-garde peut donner aux entreprises et aux 
ingénieurs l'occasion de percevoir «des saillants rentrants» dans une gamme de produits et de logiciels courants. Ils peuvent donc définir des possibilités d'amélioration et des trajectoires de solutions du casse-tête. À noter que du point de vue analytique, les forces motrices ne sont plus conceptualisées comme des causes préalables, mais comme des anticipations qui ne pourront être évaluées qu'en rétrospective. À partir d'une perspective évolutive, la sélection (rétrospective) est déterminée par la structure alors que les différences peuvent être aléatoires (Arthur, 1988; Leydesdorff et Van den Besselaar, 1998).

Troisièmement, du point de vue des anticipations, le modèle laisse place à sa base à des processus d'incertitudes et de chance. Il y aura réplication des porteurs institutionnels s'ils se sont révélés fonctionnels jusque là, mais on peut s'attendre à ce que les négociations entraînent des expériences qui pourront, elles aussi, être institutionnalisées par la suite. C'est ainsi qu'on peut préciser un modèle d'innovation par étapes. Il n'est pas nécessaire que ces étapes correspondent à la théorie de la vie utile d'un produit. Barras (1990) note, par exemple, qu'en technologie de l'information et de la communication (TIC), c'est «la vie utile d'un produit opposé » qui semble prédominer. Selon Bruckner et ses collaborateurs (1994), la création d'un créneau représente le mécanisme de blocage potentiel en cas de technologies concurrentes. Une innovation réussie change le paysage, c'est-à-dire la structure des opportunités, pour les acteurs institutionnels en cause. En retour, des changements structurels devraient changer la dynamique.

Quatrièmement, le développement de l'enseignement supérieur et l'accroissement de la recherche universitaire donnent à la société un secteur dans lequel différentes configurations peuvent se recombiner de façon systématique. Kaghan et Barett (1997) se sont servis dans ce contexte de l'expression «innovation de table de travail» pour la différencier du modèle du laboratoire (voir Etzkowitz, 1999). Des économies fondées sur la connaissance ne peuvent plus se contenter de simples mesures du profit optimal; aux fonctions d'utilité doivent correspondre des structures d'opportunité. Avec le temps, ces structures d'opportunité seront mues rétroactivement par les contingences des technologies dominantes et possibles. Un laboratoire dont les découvertes se fondent sur les connaissances est socialement disponible et peut être amélioré (Etzkowitz et Leydesdorff, 1995). Pendant que cette hélice fonctionne, le facteur capital humain se perfectionne le long des courbes d'enseignement, comme antidote au risque de chômage technologique (Pasinetti, 1981).

Cinquièmement, le modèle explique aussi pourquoi il n'est pas nécessaire de résoudre les tensions. Cela perturberait la dynamique du système qui vit des perturbations et des interactions dans les sous-systèmes. On s'attend donc à la réplication des soussystèmes. En ouvrant la boîte noire, on trouve le «Mode 1» à l'intérieur du «Mode 2» et le «Mode 2 » à l'intérieur du «Mode 1 ». Le système n'est ni intégré ni complètement différencié, mais il fonctionne au bord des différentes tractions et des intégrations locales. Avec ce modèle, on commence à comprendre pourquoi le régime global se manifeste progressivement dans les cas locaux tandis que ces derniers nous renseignent 
sur le développement global sous l'angle d'exceptions dont les réplications servent de base à de nouvelles constructions.

Les données portant sur les cas locaux permettent de préciser de façon réflexive les mécanismes de sélection négatifs. Cependant, les mécanismes de sélection demeurent des constructions. Avec le temps, l'inférence pourra être confirmée. À la fin, la fonction d'inférence réflexive fondée sur les théories disponibles ou nouvelles fait avancer le système en attirant l'attention sur des possibilités de changement.

Sixièmement, il est essentiel de relier et d'intégrer les uns dans les autres les moyens d'échange, soit les anticipations économiques (profits et croissance), les anticipations théoriques, l'évaluation de ce qui est réalisable compte tenu des contraintes institutionnelles et géographiques. Avec le temps, les hélices communiquent de façon récursive en utilisant chacune leur propre code. Jusqu'à un certain point, elles peuvent aussi échanger leurs rôles de façon réflexive. Les discours peuvent entrer en interaction aux interfaces, mais la fréquence des interactions extérieures est inférieure (du moins au départ) à leur fréquence à l'intérieur de chaque hélice. Avec le temps et avec les TIC, ces relations peuvent changer.

L'équilibre entre les relations spatiales et virtuelles dépend de la disponibilité de moyens d'échange et de leur codification. Des moyens d'échange codifiés donnent au système la possibilité de modifier la signification d'une communication (dans un autre contexte) tout en conservant sa substance (Cowan et Poray, 1997). En dépit du caractère «virtuel» de l'enveloppe, le système ne plane pas dans les airs; il est ancré dans la culture qu'il doit reproduire (Giddens, 1984). Le mécanisme de conservation n'est plus fourni, mais «en mouvement»; il est reconstruit en même temps que le système, c'està-dire comme l'une des sous-dynamiques. À mesure que la culture technologique offre des choix de recombinaisons, il est possible de reconstituer les frontières des collectivités. Le prix à payer pourrait être la perte des identités traditionnelles et l'aliénation ou l'incertitude au sujet de la viabilité de la reconstruction, mais l'option opposée à la «destruction créative» est l'accroissement du développement. Le nouveau mode de production de la connaissance génère une «transition infinie» qui redéfinit sans cesse les limites de la «frontière infinie».

\section{RÉSUMÉ}

Nous comparons le modèle à Triple hélice des relations entre l'université, l'industrie et le gouvernement avec d'autres modèles pour expliquer les transitions actuelles des systèmes de recherche dans leur contexte social. Les communications et les négociations entre les partenaires institutionnels créent une couverture réflexive qui réorganise de plus en plus les arrangements sous-jacents. Ce processus de codification interactive fonde l'économie sur la connaissance. On peut considérer le niveau institutionnel comme le mécanisme de conservation du système évolutif en développement. Historiquement, l'organisation «nationale» du système d'innovation était importante à cause de la concurrence, mais les nouvelles technologies (biotechnologie, TIC) ont entraîné une réorganisation transversale des secteurs industriels et des États nations. La recherche universitaire devient un lieu d'exploration des transitions de ces réseaux fondés sur la connaissance. 


\section{SUM MARY}

The Triple Helix of University-Industry-Government Relations is compared with alternative models for explaining the current transitions in the research system in their social contexts. Communications and negotiations between institutional partners generate a reflexive overlay that increasingly reorganizes the underlying arrangements. This process of interactive codification makes the economy knowledge-based. The institutional layer can be considered as the retention mechanism of the evolutionarily developing system. «National» organization of the systems of innovation has historically been important in determining competition, but reorganizations across industrial sectors and nation states are induced by new technologies (biotechnology, ICT). University research provides a locus of exploration in these knowledge-intensive network transitions.

\section{RESUMEN}

Nosotros comparamos el modelo de triple hélice de las relaciones entre la universidad, el gobierno y la industria con modelos alternativos para explicar las transiciones en curso en el sistema de investigación en sus contextos sociales respectivos. Las comunicaciones y las negociaciones entre socios institucionales generan una cobertura reflexiva que reorganiza cada vez más los acuerdos subyacentes. Este proceso de codificación interactiva caracteriza la economía basada en el conocimiento. La dimensión institucional puede ser considerada como un mecanismo de conservación del sistema evolutivo en desarrollo. La organización «nacional» de los sistemas de innovación ha sido históricamente importante a causa de la competición, pero las nuevas tecnologías (biotecnologías, ICT) provocaron una reorganización transversal de los sectores industriales y de los estados nacionales. La investigación universitaria ofrece un lugar de exploración de las transiciones de estas redes fundadas en el conocimiento.

\section{BIBLIOGRAPHIE}

Alchian, A. A. (1950), «Uncertainty, Evolution, and Economic Theory», Journal of Political Economy, 58, p. 211-222.

Andersen, E. S. (1994), Evolutionary Economics: Post-Schumpeterian Contributions, London, Pinter.

Arthur, W. B. (1988), «Competing technologies», in: G. Dosi, C. Freeman, R. Nelson, G. Silverberg et L. Soete (éd.), Technical Change and Economic Theory, London, Pinter, p. 590-607.

BARRAs, R. (1990), «Interactive innovation in financial and business services: The vanguard of the service revolution", Research Policy, 19, p. 215-37.

Ben David, J. et R. Collins (1966), «Social Factors in the Origins of New Science: The Case of Psychology», American Sociological Review, 3, p. 45-85.

Benner, M. et U. SAndström, «Institutionalizing the Triple Helix: Research Funding and Norms in the Academic System», Research Policy, 29, p. 291-301.

Berman, M. (1982), All That is Solid Melts into Air: The Experience of Modernity, New York, Simon and Schuster.

Braczyk, H.-J., P. Cooke et M. Heidenreich (éd.), Regional Innovation Systems, London/ Bristol PA, University College London Press.

Bruckner, E., W. Ebeling, M. A. Jiménez Montaño et A. Scharnhorst (1994), «Hyperselection and Innovation Described by a Stochastic Model of Technological Evolution», in L. Leydesdorff et P. Van den Besselaar (éd.), Evolutionary Economics and Chaos Theory: New Directions in Technology Studies, London et New York, Pinter, p. 79-90.

Bush, V. [1945] (1980), The Endless Frontier: A Report to the President, reprinted by Arno Press, New York. 
Callon, M. (1998), «An essay on Framing and Overflowing: Economic Externalities Revisited by Sociology», in The Laws of the Market, London, Macmillan, p. 244-269.

Cooke, P. (1998), «Introduction. The Origins of the Concept», in H.-J. Braczyk, P. Cooke et M. Heidenreich (éd), Regional Innovation Systems, London, Bristol PA, University College London Press, p. 2-25.

Cooke, P., M. Uranga et G. Etxeberria (1997), «Regional Innovation Systems: institutional and organizational dimensions", Research Policy, 26, p. 475-491.

Cowan, R. et D. Foray (1997), The Economics of Codification and the Diffusion of Knowledge, Maastricht, MERIT.

Cozzens, S., P. Healey, A. Rip et J. Ziman (éd.) (1990), The Research System in Transition, Boston, Kluwer Academic Publishers.

Dasgupta, P. et P. David (1994), «Towards a New Economics of Science», Research Policy, 23, p. 487-522.

David, P. A. et D. Foray (1994, «Dynamics of Competitive Technology Diffusion Through Local Network Structures: The Case of EDI Document Standards», in L. Leydesdorff et P. Van den Besselaar (Editors), Evolutionary Economics and Chaos Theory: New Directions in Technology Studies, London et New York, Pinter, p. 63-78.

Eтzкоwiтz, H., (à paraître), The Second Academic Revolution: MIT and the Rise of Entrepreneurial Science, London, Gordon and Breach.

Етzкоwiтz, H. (1999), «Bridging the Gap: The Evolution of Industry-University Links in the United States», in L. Branscomb et F. Kodama (éd.), Industrializing Knowledge: University-Industry Linkages in Japan and the United States, Cambridge MA, MIT Press.

Etzkowitz, H., M. Gulbrandsen et J. Levitt (2000), Public Venture Capital: Government Funding Sources for Technology Entrepreneurs, New York, Harcourt-Brace.

Etzkowitz, H. et L. Leydesdorff (éd.) (1997), Universities in the Global Economy: A Triple Helix of University-Industry-Government Relations, London, Cassell Academic.

Etzkowitz, H. et L. LeYdesdorfF (1995), «The Triple Helix--University-Industry-Government Relations: A Laboratory for Knowledge-Based Economic Development», EASST Review, vol. 14, nº 1, p. 14-19.

Freeman, C. (1982), The Economics of Industrial Innovation, London, Pinter.

Freeman, C. et C. Perez (1988), «Structural Crises of Adjustment, Business Cycles and Investment Behaviour", in G. Dosi, C. Freeman, R. Nelson, G. Silverberg, et L. Soete (éd.), Technical Change and Economic Theory, London, Pinter, p. 38-66.

Frenken, K. (2000), «A Complexity Approach to Innovation Networks. The Case of the Aircraft Industry (1909-1997)», Research Policy, 29, p. 257-272.

Frenken, K. et L. Leydesdorff (2000), «Scaling Trajectories in Civil Aircraft (1913-1997)», Research Policy, 29, p. 331-348

Gibbons, M., C. Limoges, H. Nowotny, S. Schwartzman, P. Scott et M. Trow (1994), The New Production of Knowledge: the Dynamics of Science and Research in Contemporary Societies, London, Sage.

Giddens, A. (1984), The Constitution of Society, Cambridge, Polity Press.

Godin, B. (1998), «Writing Performative History: Is This a New Atlantis?» Social Studies of Science, vol. 38, $\mathrm{n}^{\mathrm{o}} 3$, p. 465-483.

Godin, B. et Y. Gingras, «The Place of Universities in the System of Knowledge Production», Research Policy, 29, p. 273-278.

Government-University-Industry Research Roundtable GUIRR (1998), National Science and Technology Strategies in a Global Context. Report of an International Symposium, Washington D.C., National Academy Press, $<$ http://bob.nap.edu/readingroom/books/nst/>.

Gustin, B. (1975), The Emergence of the German chemical profession, 1790-1867. Ph.D. dissertation, University of Chicago.

Hughes, T. P. (1983), Networks of Power: Electrification of Western Society 1880-1930, Baltimore MD, Johns Hopkins University Press.

Kaghan, W. N. et G. B. Barnett (1997), «The Desktop Model of Innovation», in H. Etzkowitz et L. Leydesdorff (éd.), Universities in the Global Economy: A Triple Helix of University-Industry-Government Relations, London, Cassell Academic, p. 71-81. 
Kampmann, C., C. Haxholdt, E. Mosekilde et J. D. Sterman (1994), «Entrainment in a Disaggregated Long-Wave Model», in L. Leydesdorff et P. Van den Besselaar (éd.), Evolutionary Economics and Chaos Theory: New Directions in Technology Studies, London et New York, Pinter, p. 109-124.

LAngton, C. G. (éd.) (1989), Artificial Life, Redwood City, CA, Addison Wesley.

LeydesdorfF, L. (1997), «The Non-linear Dynamics of Sociological Reflections», International Sociology, 12, p. $25-45$.

LEYDESDORFF, L. (1995), The Challenge of Scientometrics: the development, measurement, and self-organization of scientific communications, Leiden University, Leiden: DSWO Press.

Leydesdorff, L. et H. Etzkowitz (1998), «The Triple Helix as a model for innovation studies», Science and Public Policy, vol. 25, nº 3, p. 195-203.

LeydesdorfF, L. et H. Eтzкоwitz (1996), «Emergence of a Triple Helix of University-Industry-Government Relations», Science and Public Policy, 23, p. 279-286.

LeydesdorfF, L. et N. Oomes (1999), «Is the European Monetary System Converging to Integration?», Social Science Information, vol. $38, \mathrm{n}^{\circ}$ 1, p. 57-86.

LeydesdorfF, L. et P. van den Besselaar (1998), «Competing Technologies: Lock-ins and Lock-outs», in D. M. Dubois (éd.), Computing Anticipatory Systems, Proceedings of the American Institute of Physics, 437, Woodbury, NY, American Institute of Physics, p. 309-323.

LeydesdorfF, L. et P. VAn den BesselaAr (1997), «Scientometrics and Communication Theory: Towards Theoretically Informed Indicators", Scientometrics, 38, p. 155-74.

Luhmann, N. (1984), Soziale Systeme. Grundriß einer allgemeinen Theorie, Frankfurt a. M., Suhrkamp, [Social Systems, Stanford, Stanford University Press, 1995].

LundvalL, B.-Å. (éd.) (1992), National Systems of Innovation, London, Pinter.

Lundvall, B.-Å. (1988), «Innovation as an Interactive Process: from User-producer Interaction to the National System of Innovation», in G. Dosi, C. Freeman, R. Nelson, G. Silverberg et L. Soete (éd.), Technical Change and Economic Theory, London, Pinter, p. 349-369.

MacLane, S. (1996), «Should Universities Imitate Industry?», American Scientist, vol. 84, nº 6, p. 520-521.

Maturana, H. R. (1978), «Biology of Language: The Epistemology of Reality», in G. A. Miller et E. Lenneberg (éd.), Psychology and Biology of Language and Thought. Essays in Honor of Eric Lenneberg, New York, Academic Press, p. 27-63.

McKelvey, M. D. (1996), Evolutionary Innovations: The Business of Biotechnology, Oxford, Oxford University Press.

Merton, R. K. (1942), «Science and Technology in a Democratic Order», Journal of Legal and Political Sociology, 1, p. 115-26.

Merton, R. K. (1938), Science, Technology and Society in Seventeenth Century England, Burges, Sainte Catherine Press.

Mills, C. W. (1958), The Power Elite, New York, Oxford University Press.

Mowery, D. C. et N. Rosenberg (1979), «The Influence of Market Demand upon Innovation: a Critical Review of Some Empirical Studies», Research Policy, 8, p. 102-153.

Nelson, R. R. (1994), «Economic Growth via the Coevolution of Technology and Institutions», in L. Leydesdorff et P. Van den Besselaar (éd.), Evolutionary Economics and Chaos Theory: New Directions in Technology Studies, London et New York, p. 21-32.

Nelson, R. R. (éd.) (1993), National Innovation Systems: A comparative study, New York, Oxford University Press.

Nelson, R. R. (éd.) (1982), Government and Technical Progress: a cross-industry analysis, New York, Pergamon. Nelson, R. et S. Winter (1975), «Growth Theory from an Evolutionary Perspective: The Differential Productivity Growth Puzzle», American Economic Review, 65, 338.

Nelson, R. R. et S. G. Winter (1982), An Evolutionary Theory of Economic Change, Cambridge, MA, Belknap Press.

Neurath, O., R. Carnap et H. Hahn (1929), Wissenschaftliche Weltauffassung — Der Wiener Kreis, Vienna, Veröffentlichungen des Vereins Ernst Mach. 
oECD (1980), Technical Change and Economic Policy, Paris, OECD.

Pasinetti, L. (1981), Structural Change and Economic Growth, Cambridge, Cambridge University Press.

Pavits, K. (1984), «Sectoral Patterns of Technical Change: towards a Theory and a Taxonomy», Research Policy, 13, p. 343-73.

Riba-Vilanova, M. et L. Leydesdorff (1999), A Triple Helix of University-Industry-Government Relations at the Regional Level: The Case of Catalonia, Paper presented at the European workshop on Regional Innovation Systems, San Sebastian/Donostia, octobre 1999.

Rip, A. et B. Van der Meulen (1996), «The Post-modern Research System», Science and Public Policy, vol. $23, n^{\circ} 6$, p. 343-352.

Rosenberg, N. et R. R. Nelson (1994), «American Universities and Technical Advance in Industry», Research Policy, 23, p. 323-348.

Rothwell, R. et W. Zegveld (1981), Industrial Innovation and Public Policy, London, Pinter.

SÁвато, J. (1975), El pensamiento latinoamericano en la problemática ciencia-technología-desarrollodependencia, Buenos Aires, Paidós.

Sábato, J. et M. Mackenzi (1982), La producción de technología. Autónoma o transnacional, Mexico, Nueva Imagen.

SAXENian, A. (1994), Regional advantage: Culture and competition in Silicon Valley and Route 128, Cambridge MA/ London, Harvard University Press.

Schumpeter, J. (1966), Invention and Economic Growth, Cambridge, MA, Harvard University Press.

Schumpeter, J. [1939] (1964), Business Cycles: A Theoretical, Historical and Statistical Analysis of Capitalist Process, New York, McGraw-Hill.

Sobel, D. (1995), Longitude, Harmondsworth, Penguin.

Storr, R. (1953), The Beginnings of Graduate Education in America, Chicago, University of Chicago Press. Weingart, P. (1997), "From "Finalization" to "Mode 2": old wine in new bottles?», Social Science Information, vol. $36, \mathrm{n}^{\circ} 4$, p. 591-613.

Ziman, J. (1994), Prometheus Bound: Science in a Dynamic Steady State, Cambridge, Cambridge University Press. 
FIGURE 1

Modèle de relations étatiques entre l'université, l'industrie et le gouvernement

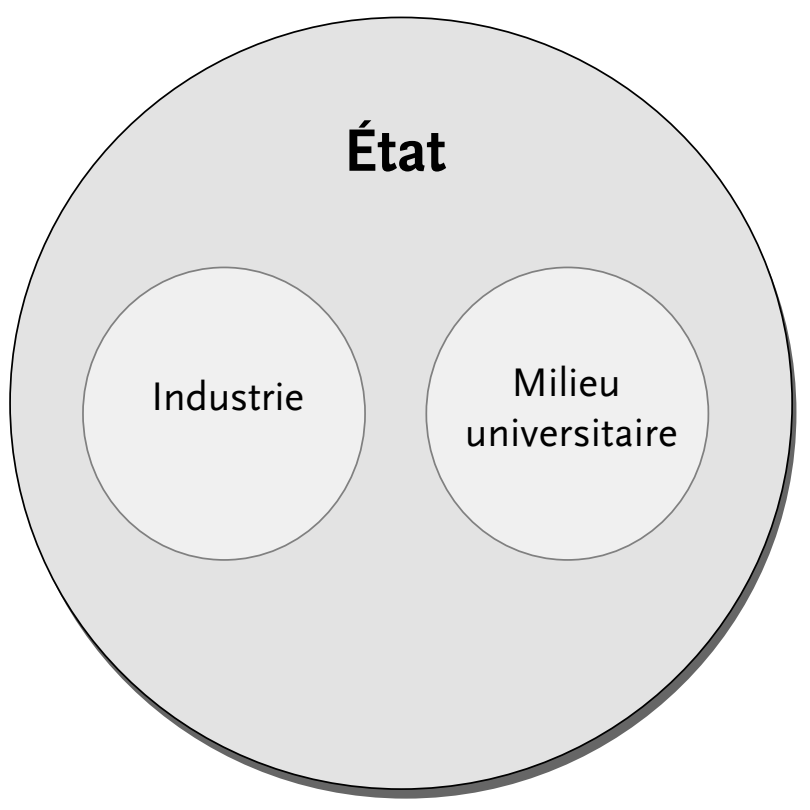

FIGURE 2

Modèle de relations de laisser-faire entre l'université, l'industrie et le gouvernement

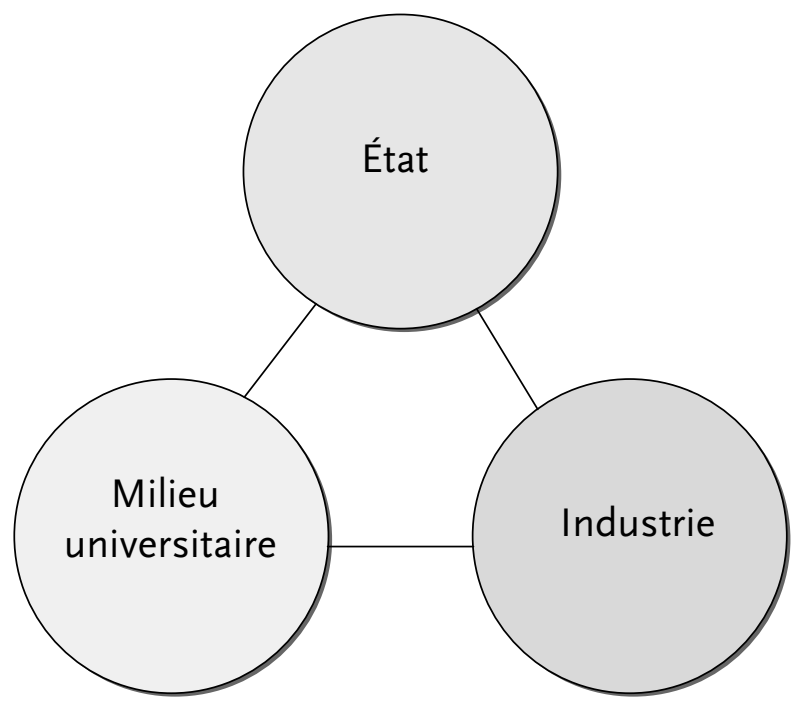


FIGURE 3

Modèle à triple hélice entre l'université, l'industrie et le gouvernement

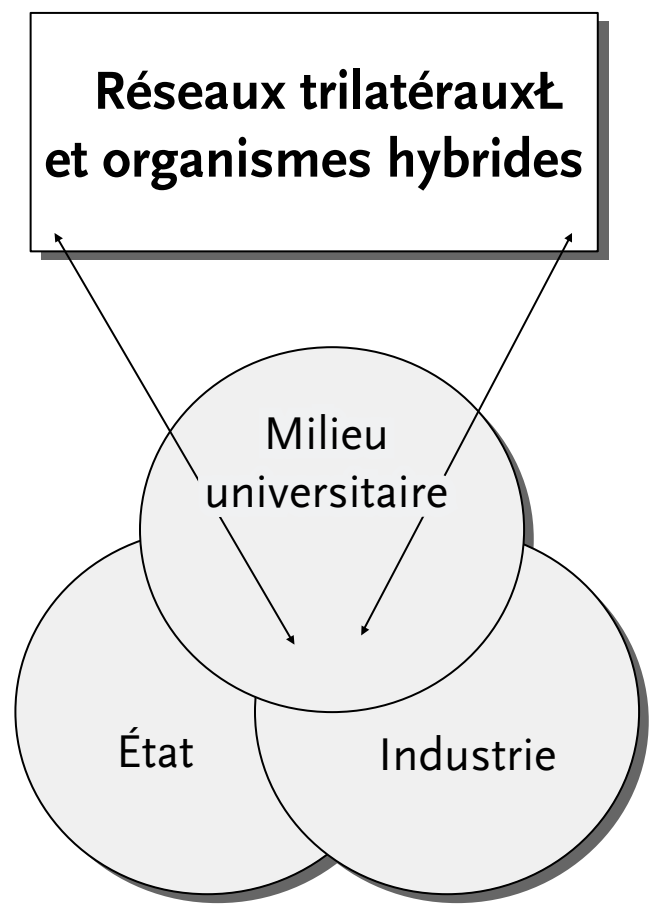

